\title{
Adaptive Neuro-Fuzzy Modeling of Battery Residual Capacity for Electric Vehicles
}

\author{
W. X. Shen, Student Member, IEEE, C. C. Chan, Fellow, IEEE, E. W. C. Lo, Member, IEEE, and \\ K. T. Chau, Member, IEEE
}

\begin{abstract}
This paper proposes and implements a new method for the estimation of the battery residual capacity (BRC) for electric vehicles (EVs). The key of the proposed method is to model the $\mathrm{EV}$ battery by using the adaptive neuro-fuzzy inference system. Different operating profiles of the $\mathrm{EV}$ battery are investigated, including the constant current discharge and the random current discharge as well as the standard EV driving cycles in Europe, the U.S., and Japan. The estimated BRCs are directly compared with the actual BRCs, verifying the accuracy and effectiveness of the proposed modeling method. Moreover, this method can be easily implemented by a low-cost microcontroller and can readily be extended to the estimation of the BRC for other types of $\mathrm{EV}$ batteries.
\end{abstract}

Index Terms-Adaptive neuro-fuzzy inference system, battery modeling, battery residual capacity, electric vehicles.

\section{INTRODUCTION}

W ITH ever-increasing concerns over environmental protection and energy conservation, research and development on various electric vehicle (EV) technologies are being actively conducted [1], [2]. However, the application technology of EV batteries, namely, the driving range indicator, cannot keep pace with the development of other EV technologies. At present and in the near future, batteries have been identified to be the major energy source for EVs because of their technological maturity and reasonable cost. Therefore, the key to the development of the driving range indicator for EVs is to accurately estimate the battery residual capacity (BRC) [3].

Conventionally, the estimation of the BRC can be categorized into three groups. The first group is based on the empirical formula [4]-[7] and the mathematical model (or the equivalent electric circuit) [8]-[10] through the analysis of battery characteristics of the constant current discharge. The second group is based on the coulometric measurement in which the accumulated error is corrected by the stabilized open-circuit voltage (OCV) [11]. The third group is based on the impedance measurement [12], [13]. For the first group, the parameters of the methods are generally obtained only from the steady state, which cannot reflect the dynamic behavior of the battery in EVs. Moreover, these methods highly depend on a particular type of battery. It is not easy to extend them from one type of battery to another. For the second group, the stabilized OCV refers to

Manuscript received February 27, 2001; revised December 25, 2001. Abstract published on the Internet March 7, 2002. This work was supported in part by an RGC Research Grant from the Hong Kong Research Grants Council and a CRCG Research Grant from the University of Hong Kong.

The authors are with the Department of Electrical and Electronic Engineering, The University of Hong Kong, Hong Kong (e-mail: ktchau@eee.hku.hk).

Publisher Item Identifier S 0278-0046(02)04915-8. the battery terminal voltage measured after the battery is sufficiently rested, for instance, $30 \mathrm{~min}$ [11]. However, practical EV operation may not have much chance to stop for such a long period that the stabilized OCV can be measured. For the third group, a small amplitude signal of alternative current is injected into the battery for the measurement of the internal impedance. As a result, the impedance obtained does not include the dynamic characteristic of the large discharge current, which is often exposed to the EV battery. Furthermore, the necessary equipment to carry out such impedance measurement is too expensive and bulky for EVs.

Different from the aforementioned methods, the application of the artificial neural network (ANN) to the estimation of the BRC under variable current discharge [14], [15] and constant current discharge [16], [17] provides a tool to deal with the above difficulties. This is due to two key features of the ANN. First, the ANN does not rely on the explicitly expressed relationship between input variables and the BRC. When using the ANN for the BRC estimation, one needs to only consider the selection of variables as the ANN inputs. The relationship between the input variables and the BRC is formulated by a training process, avoiding those difficulties in the modeling process. Second, the adaptive algorithm is another attractive feature of the ANN. An updated training data set can be used to retrain the ANN so that the ANN can adapt the change of the BRC in the most recent conditions. On the other hand, the fuzzy logic is also explored in the estimation of the BRC [18]. This is due to the fact that the fuzzy logic can handle uncertainties and imprecision in the real battery system. Moreover, the parameters of the fuzzy system have clear physical meanings so that rule-based and linguistic information can be incorporated into the fuzzy system.

The ANN for the estimation of the BRC cannot provide heuristic knowledge of the battery on the BRC estimation process because of its black-box approach. On the other hand, fuzzy logic is a tool that can easily implement and utilize heuristic reasoning, but it is generally difficult to provide exact solutions. With the integrated synergy of the ANN and the fuzzy logic, the estimation of the BRC using adaptive neuro-fuzzy inference system (ANFIS) can function to provide more accurate solutions under different operating conditions and also a better understanding of the estimation process. Therefore, the purpose of this paper is to develop a new estimation approach of the BRC by using the ANFIS. The idea behind the fusion of the ANN and fuzzy logic is to use the learning ability of the ANN to implement and automate the fuzzy system which utilizes the high-level human-like reasoning capability. To facilitate the application to EVs, the estimation of the BRC 
Layer 1 Layer 2 Layer 3 Layer 4 Layer 5

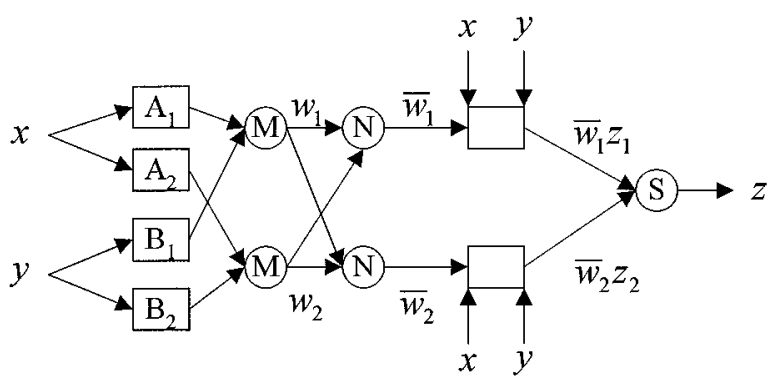

Fig. 1. ANFIS architecture.

is only based on those measurable variables, namely, the battery terminal voltage, the discharge current, the discharged capacity, and the battery surface temperature. Once the ANFIS is formulated, the method can be easily implemented by a low-cost microcontroller for the BRC estimation in EVs.

\section{ANFIS MODELING}

\section{A. ANFIS Architecture}

The ANFIS is a fuzzy Sugeno model put in the framework of adaptive systems to facilitate learning and adaptation [19]. Such a framework makes the ANFIS modeling more systematic and less reliant on expert knowledge. To present the ANFIS architecture, the following two fuzzy if-then rules based on a first-order Sugeno model are considered:

- Rule 1: if ( $x$ is $\left.A_{1}\right)$ and ( $y$ is $\left.B_{1}\right)$ then $\left(z_{1}=p_{1} x+q_{1} y+r_{1}\right)$

- Rule 2: if ( $x$ is $\left.A_{2}\right)$ and ( $y$ is $\left.B_{2}\right)$ then $\left(z_{2}=p_{2} x+q_{2} y+r_{2}\right)$ where $x$ and $y$ are the inputs, $A_{i}$ and $B_{i}$ are the fuzzy sets, $z_{i}$ are the outputs within the fuzzy region specified by the fuzzy rule, and $p_{i}, q_{i}$, and $r_{i}$ are the design parameters to be determined during the training process. Thus, the possible ANFIS architecture to implement these two rules is shown in Fig. 1. Note that a circle indicates a fixed node while a square indicates an adaptive node (the parameters are changed during adaptation or training).

In this five-layered architecture, all the nodes in Layer 1 are adaptive nodes. The output of each node $i$ is the degree of membership of the input represented by

$$
O_{i}^{1}= \begin{cases}\mu_{A_{i}}(x), & i=1,2 \\ \mu_{B_{i-2}}(y), & i=3,4\end{cases}
$$

where $\mu_{A_{i}}(x), \mu_{B_{i-2}}(y)$ can be adopted by any fuzzy membership function (MF). For example, if the bell MF is used, it yields

$$
\mu_{A_{i}}(x)=\frac{1}{1+\left(\frac{x-c_{i}}{a_{i}}\right)^{2 b_{i}}}
$$

where $a_{i}, b_{i}$, and $c_{i}$ are the parameters that change the shape of the MF

In Layer 2, each node is a fixed node. They are labeled " $M$ " to indicate that they play the role of a simple multiplier. The outputs of these nodes are given by:

$$
O_{i}^{2}=w_{i}=\mu_{A_{i}}(x) \mu_{B_{i}}(y), \quad i=1,2 .
$$

The output of each node in this layer represents the firing strength $w_{i}$ of the rule.
Each node in the Layer 3 is also a fixed node. They are labeled " $\mathrm{N}$ " to indicate that they perform a normalization of the firing strength from the previous layer. The output of each node in this layer is given by

$$
O_{i}^{3}=\bar{w}_{i}=\frac{w_{i}}{w_{1}+w_{2}}, \quad i=1,2
$$

where $\bar{w}_{i}$ refers to the normalized firing strength.

In Layer 4, each node is an adaptive node. The output of each node in this layer is simply the product of the normalized firing strength and a first-order polynomial (for the first-order Sugeno model)

$$
O_{i}^{4}=\bar{w}_{i} z_{i}=\bar{w}_{i}\left(p_{i} x+q_{i} y+r_{i}\right), \quad i=1,2 .
$$

Only one node labeled " $\mathrm{S}$ " is in Layer 5. It performs the function of a simple summer. The output of this single node is given by

$$
O_{1}^{5}=z=\sum_{i=1}^{2} \bar{w}_{i} z_{i}=\frac{\sum_{i=1}^{2} \bar{w}_{i} z_{i}}{\sum_{i=1}^{2} w_{i}} .
$$

From this ANFIS architecture, it is observed that there are two adaptive layers (Layer 1 and Layer 4). Layer 1 has three modifiable parameters $\left(a_{i}, b_{i}, c_{i}\right)$ related to the input MFs. These parameters are called premise parameters. Layer 4 also has three modifiable parameters $\left(p_{i}, q_{i}, r_{i}\right)$ pertaining to the first-order polynomial. These parameters are called consequent parameters. The task of the learning algorithm for this architecture is to tune all the modifiable parameters to make the ANFIS output match the training data. Note that if the parameters $a_{i}, b_{i}$, and $c_{i}$ of the bell MFs are fixed, the output of the ANFIS becomes

$$
\begin{aligned}
z= & \bar{w}_{1} z_{1}+\bar{w}_{2} z_{2} \\
= & \bar{w}_{1}\left(p_{1} x+q_{1} y+r_{1}\right)+\bar{w}_{2}\left(p_{2} x+q_{2} y+r_{2}\right) \\
= & \left(\bar{w}_{1} x\right) p_{1}+\left(\bar{w}_{1} y\right) q+\left(\bar{w}_{1}\right) r_{1}+\left(\bar{w}_{2} x\right) p_{2} \\
& +\left(\bar{w}_{2} y\right) q_{2}+\left(\bar{w}_{2}\right) r_{2}
\end{aligned}
$$

which is a linear combination of the modifiable parameters $p_{i}$, $q_{i}$, and $r_{i}$. Therefore, the least-square method (LSM) can easily identify the optimal values for these parameters. However, if the MFs are not fixed and are allowed to vary, then the search space becomes larger and, consequently, the convergence of the training algorithm becomes slower. In such case, a hybrid learning algorithm combining the LSM with the gradient descent is adopted, which is composed of a forward pass and a backward pass. In the forward pass, the premise parameters are fixed and the vector of the outputs $Z$, corresponding to $m$ input samples $\left(x_{k}, y_{k}: k=1, \ldots, m\right)$, can be deduced from (7) and written in the following matrix form:

$$
Z=W \Phi
$$

where $W=\left[\left(\bar{w}_{i} x_{j}, \bar{w}_{i} y_{j}, \bar{w}_{i}: i=1,2\right): j=1, \ldots, m\right]^{T}$ is the coefficient matrix and $\Phi=\left[p_{i}, q_{i}, r_{i},: i=1,2\right]^{T}$ is the vector of the consequent parameters. Then, (8) is solved using the LSM to determine the consequent parameters. Once the optimal consequent parameters are found, the backward pass stage 


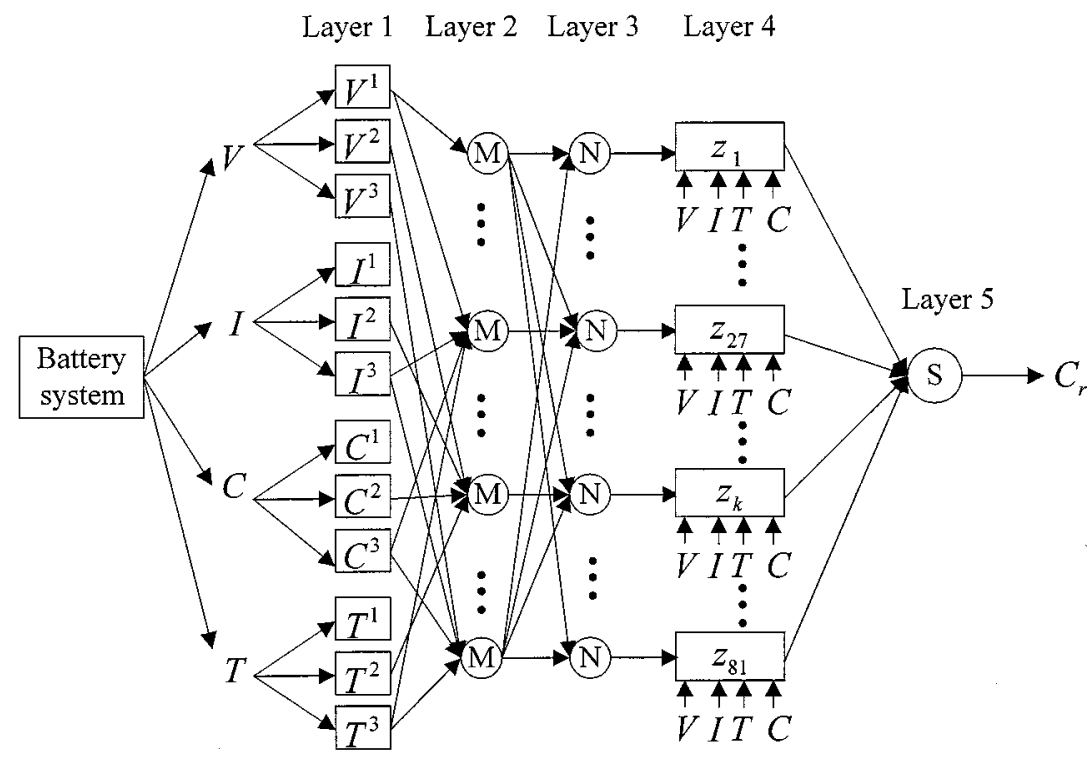

Fig. 2. ANFIS model for BRC estimation.

starts. In this stage, the gradient descent is used to optimally adjust the premise parameters corresponding to the fuzzy sets in the input domain. The output of the ANFIS is calculated by fixing the consequent parameters to the values found in the forward pass. By comparing the estimated output with the actual output, the output error of the ANFIS is then propagated from the output to the input to adapt the premise parameters using the standard backpropagation algorithm. It has been proven that the ANFIS can be used as a universal approximator [20] and the hybrid learning algorithm is highly efficient in training this ANFIS [21].

\section{B. ANFIS Model Formulation}

The ANFIS model for the estimation of the BRC is a FIS implemented on an adaptive ANN structure. The battery terminal voltage $V$, the discharge current $I$, the discharged capacity $C$, and the battery surface temperature $T$ are used as the inputs of the ANFIS model while the BRC value $C_{r}$ is used as the single output of the ANFIS model.

The universes of discourse of the battery terminal voltage, the discharge current, the discharged capacity, and the battery surface temperature are, respectively, defined as

$$
\left\{\begin{array}{l}
\mathbf{V}=\left\{V \mid V_{l} \leq V \leq V_{u}\right\} \\
\mathbf{I}=\left\{I \mid I_{l} \leq I \leq I_{u}\right\} \\
\mathbf{C}=\left\{C \mid 0 \leq C \leq C_{u}\right\} \\
\mathbf{T}=\left\{T \mid T_{l} \leq T \leq T_{u}\right\}
\end{array}\right.
$$

where $V_{l}, V_{u}, I_{l}, I_{u}, T_{l}$, and $T_{u}$ are the constants representing the upper and lower bounds of the feasible operating ranges of the battery terminal voltage, the discharge current, and the battery surface temperature, and $C_{u}$ is the upper limit of the feasible operating range of the discharged capacity under consideration. Three fuzzy sets are defined on each of the input spaces, corresponding to low, medium, and high for each variable, and labeled $V^{k}, I^{k}, C^{k}$, and $T^{k}$, respectively, with $k=1,2,3$. The input space $\mathbf{X}$ is defined as the Cartesian product spaces of the battery terminal voltage, the discharge current, the discharged capacity, and the battery surface temperature

$$
\mathbf{X}=\mathbf{V} \times \mathbf{I} \times \mathbf{C} \times \mathbf{T} .
$$

The output of the ANFIS model is the BRC, which refers to the percentage of the battery available capacity (BAC). Thus, the output space $\mathbf{Y}$ can be defined as

$$
\mathbf{Y}=\mathbf{C}_{\mathbf{r}}=\left\{C_{r} \mid 0 \leq C_{r} \leq 1\right\}
$$

The BRC estimation process can be viewed as a mapping from the input space into the output space, which maps the battery terminal voltage, the discharge current, the discharged capacity, and the battery surface temperature to the BRC. Fig. 2 shows this ANFIS model for the BRC estimation in EVs.

\section{EXPERIMENT}

Battery testing plays an important role in evaluating the performance of batteries, especially for those batteries used in EVs. Fig. 3 shows the battery evaluation and testing system (BETS), which was built at the International Research Center for Electric Vehicles, The University of Hong Kong, Hong Kong. This system consists of the following four main parts:

- programmable charger in which almost any charging algorithms can be performed;

- programmable electronic load in which flexible and variable discharge regimes can be designed, such as the constant current discharge, the constant power discharge, the constant resistance discharge, and the variable current discharge according to EV driving cycles;

- temperature-controlled chamber in which batteries can be tested under any predefined air temperature over the range from $-20{ }^{\circ} \mathrm{C}$ to $50{ }^{\circ} \mathrm{C}$;

- data acquisition subsystem in which the sampling time can be preset as in the order of seconds, minutes, or hours depending on the requirements of users. 


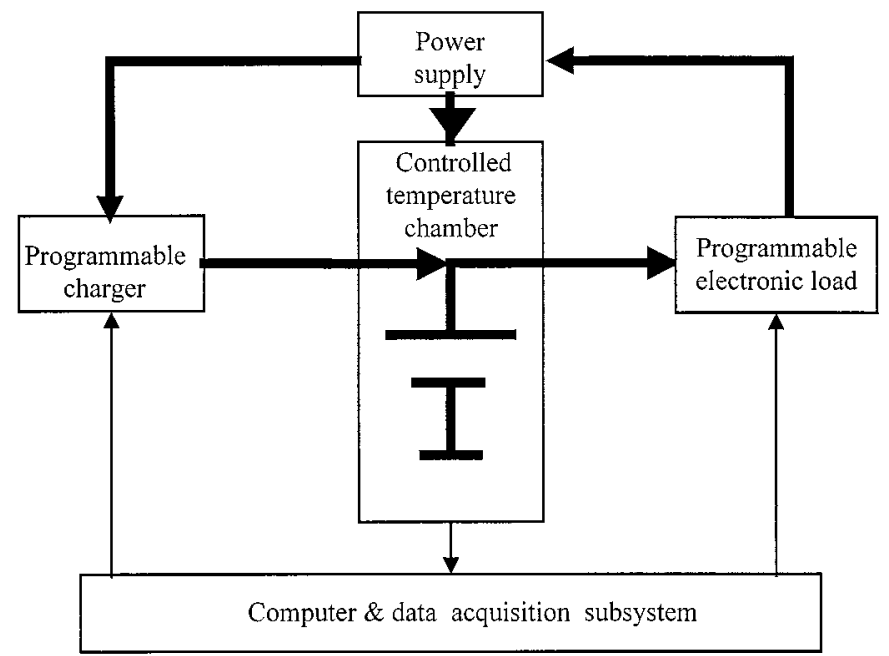

Fig. 3. Battery evaluation and testing system.

The lead acid battery with the nominal voltage of $12 \mathrm{~V}$ is used for testing, whose rated capacity is $40 \mathrm{Ah}$ at the 20-h discharge rate, namely, $C_{20}=40 \mathrm{Ah}$. However, the rated capacity for EVs is only a reference value which cannot be considered as the actual BAC because the discharge current in EVs is generally much higher than that corresponding to the rated capacity. As a consequence, the BAC in EVs is generally much lower than the rated capacity. Since the BAC varies with the change of discharge current, it is defined as the quantity of electricity that can be delivered by a fully charged battery at a certain discharge current and temperature until the specified cutoff voltage is reached. Mathematically, it can be written as

$$
C_{a}=\left.f(V, I, T)\right|_{V=V_{\text {off }}}
$$

where $C_{a}$ is the BAC, $V$ is the instantaneous terminal voltage, $I$ is the discharge current, $T$ is the battery surface temperature, and $V_{\text {off }}$ is the specified cutoff voltage. In accordance with this definition, many experiments that simulate the battery operated in EVs are conducted on the BETS. They include the constant current discharge (CCD) and the random current discharge (RCD) as well as the variable current discharge corresponding to the European reference driving cycle (ECE), the U.S. Federal urban driving schedule (FUDS), and the Japanese mode 10.15 (JM10.15). The corresponding experimental data are automatically recorded, with each row representing the battery terminal voltage, the discharge current, the discharged capacity, and the battery surface temperature as well as the BRC.

\section{DATA PROcessing}

\section{A. Filtering Data}

From the measured data, it is observed that the battery terminal voltage varies significantly with the rapid change of the discharge current, while the BRC decreases monotonically with the increase of the discharged capacity. Thus, filtering is necessary to extract the essential features of the battery for the BRC

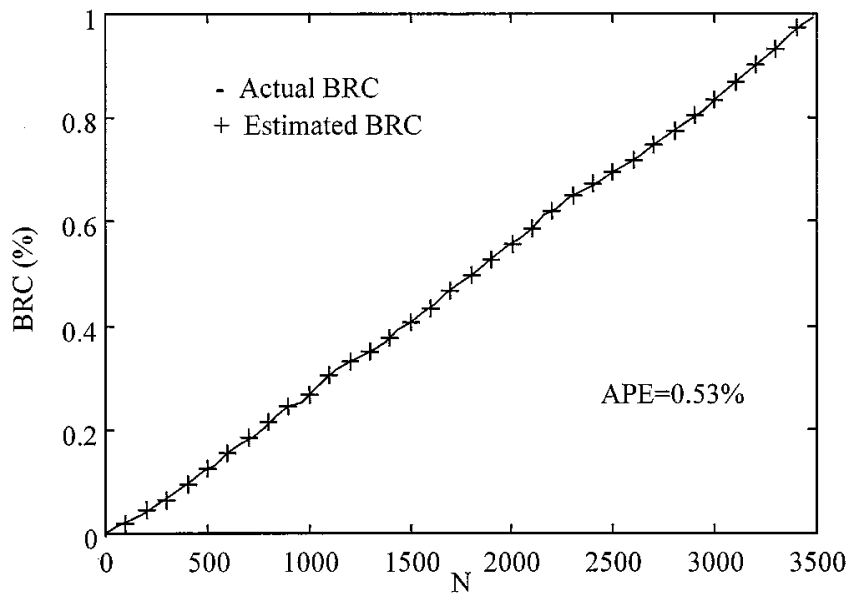

Fig. 4. Comparison between actual BRC and estimated BRC for training data.

estimation. In this study, a simple $r$-mean filter is used for convenient realization, which is given by

$$
\begin{aligned}
\overline{X Y}_{1} & =X Y_{1}, \ldots, \overline{X Y}_{(r-1)}=X Y_{(r-1)} \\
\overline{X Y}_{(r+n)} & =\frac{1}{r} \sum_{i=1}^{r} X Y_{(i+n)}, \quad n=0,1,2, \ldots
\end{aligned}
$$

where $X Y_{(i+n)}$ is the original measured data and $\overline{X Y}_{(i+n)}$ is the filtered data. The step $r$ can be selected depending on the changing frequency of the battery terminal voltage. In general, the higher the frequency, the larger the step $r$ is selected.

\section{B. Selecting Data}

The ANFIS model can make the estimation of the BRC only based on the way that it is learned from the experimental data. Thus, the selection of the training data from the numerous filtered data significantly affects the estimation of the BRC. One purpose of the data selection is to remove those data that are correlated with other data, namely, when the experimental data including the battery terminal voltage, the discharge current, the discharged capacity, and the battery surface temperature have no observable change within several samples, only one of them needs to be included in the training data set. Another purpose of the data selection is to keep the training data set as small as possible to reduce the training time for model construction. Similar to the input selection of the ANFIS proposed in [22], the following procedure is used to select the training data.

Step 1) Set the value $r$ and use (13) to filter the measured data for each test.

Step 2) Put all the filtered data into one data set and form the whole data set.

Step 3) Assign the initial size of the training data set as small as $5 \%$ of the whole data set.

Step 4) Select the training data from the whole data set randomly.

Step 5) Train the ANFIS model using the training data and then stop right after the first epoch is finished. 


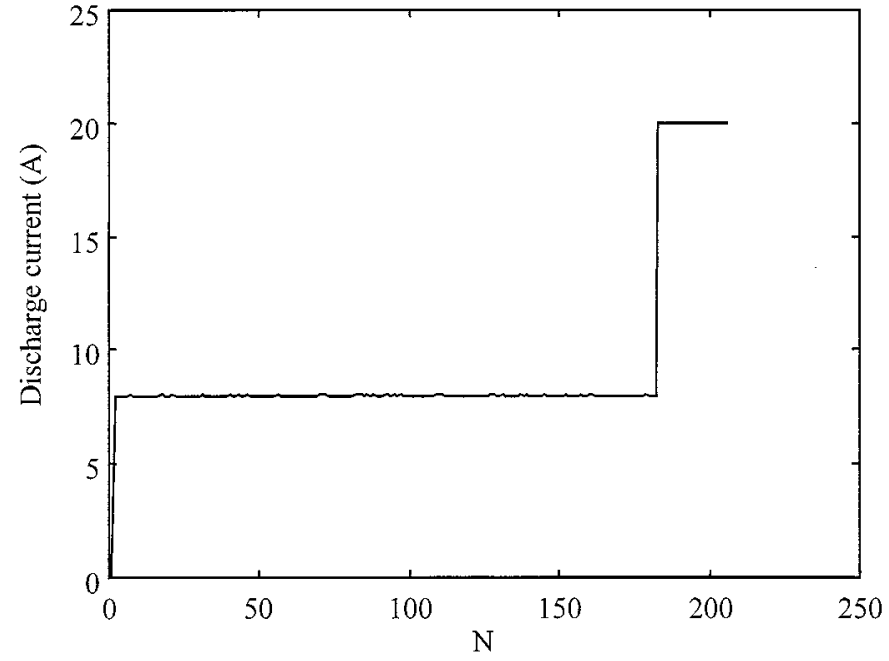

(a)

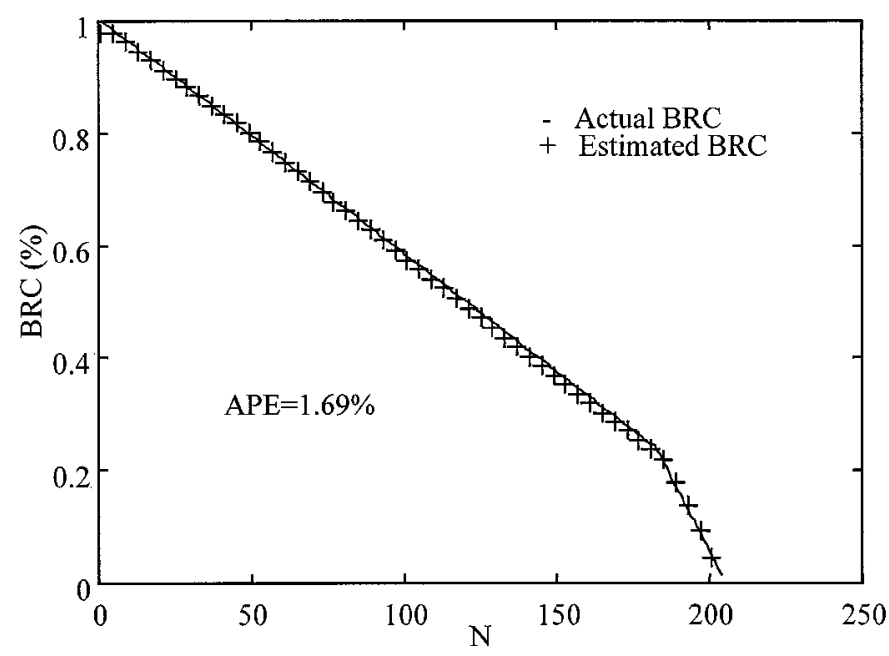

(b)

Fig. 5. Comparison between actual BRC and estimated BRC for CCD operation. (a) CCD profile. (b) BRC profiles.

Step 6) Check whether the predefined error criterion for the whole data set is achieved for the trained ANFIS model. If it is achieved, the selection procedure stops and the corresponding data is recorded as the training data set. Otherwise, go to Step 7).

Step 7) Increase the size of the training data set by $1 \%$, and then go back to Step 4).

As described previously, the ANFIS employs an efficient hybrid learning algorithm that combines the gradient descent and the LSM. In this learning algorithm, the LSM is the major driving force that leads to fast training while the gradient descent serves to slowly change the underlying MFs that generate the basis functions for the LSM. Since the LSM is computationally efficient, various combinations of the data can be selected to train the ANFIS model with a single application of the LSM, namely, right after the first epoch of training. As a result, one data set with the smallest root-mean-squared error will be selected as the training data set and proceeded for

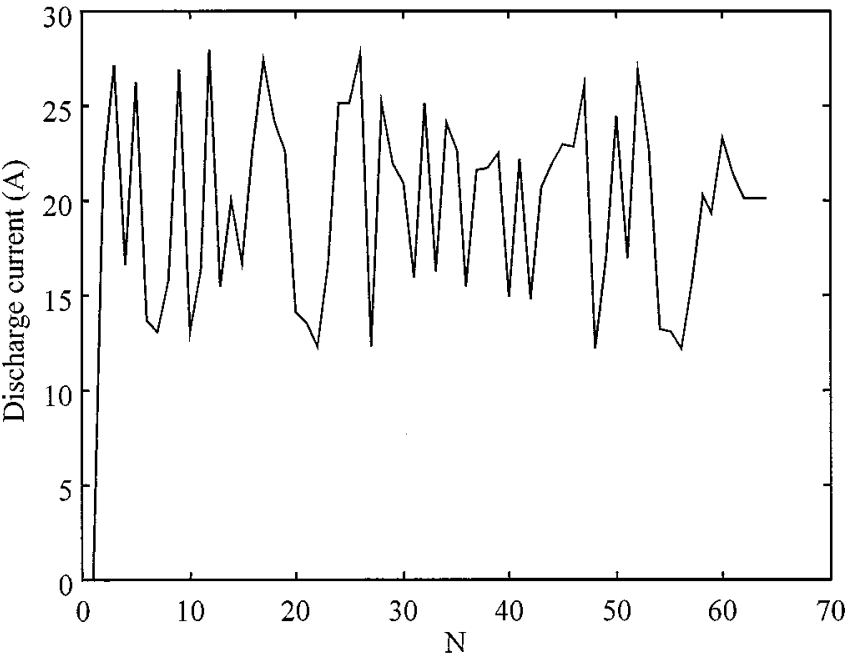

(a)

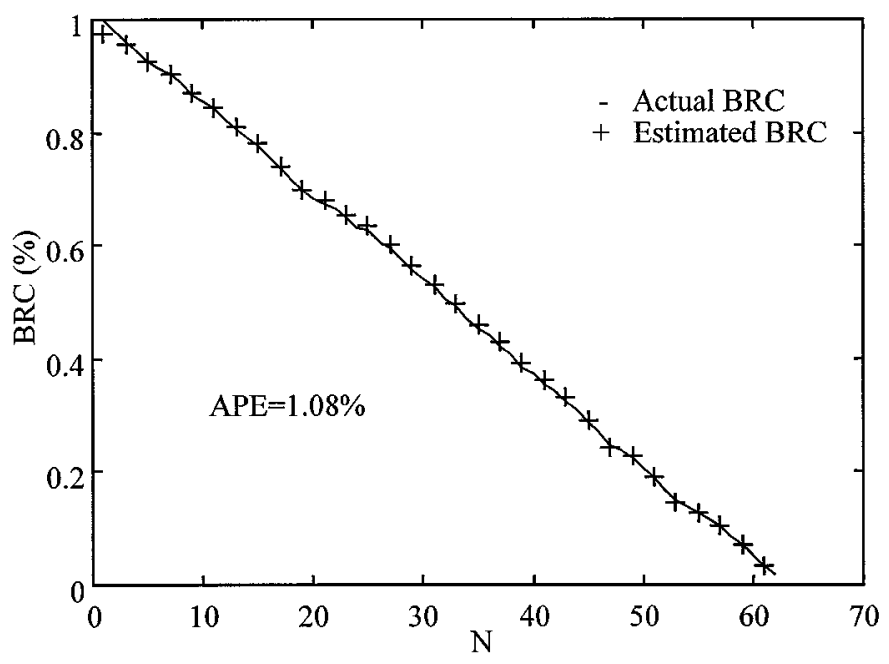

(b)

Fig. 6. Comparison between actual BRC and estimated BRC for RCD operation. (a) RCD profile. (b) BRC profiles

further training. The whole data set composed of 17 data files corresponding to 17 tests is involved in the selection procedure with 26822 records. Finally, the training data set contains 3500 data records, or $13 \%$ of the total number of the data records in the whole data set.

\section{EVAlUATION AND RESUlts}

After the data processing is finished, the training data set is used to train the ANFIS model further with the hybird learning algorithm, while the whole data set is used to verify the accuracy of the estimation of the BRC. To allow for comparison, the average percentage error (APE) is adopted. It is defined as

$$
\mathrm{APE}=\frac{1}{N} \sum_{j=1}^{N} \frac{\left|C_{r a}(j)-C_{r e}(j)\right|}{\left|C_{r a}(j)\right|} 100 \%
$$

where $N$ is the number of the training data set or the data set for each test, and $C_{r e}$ and $C_{r a}$ refer to the estimated BRC from the 


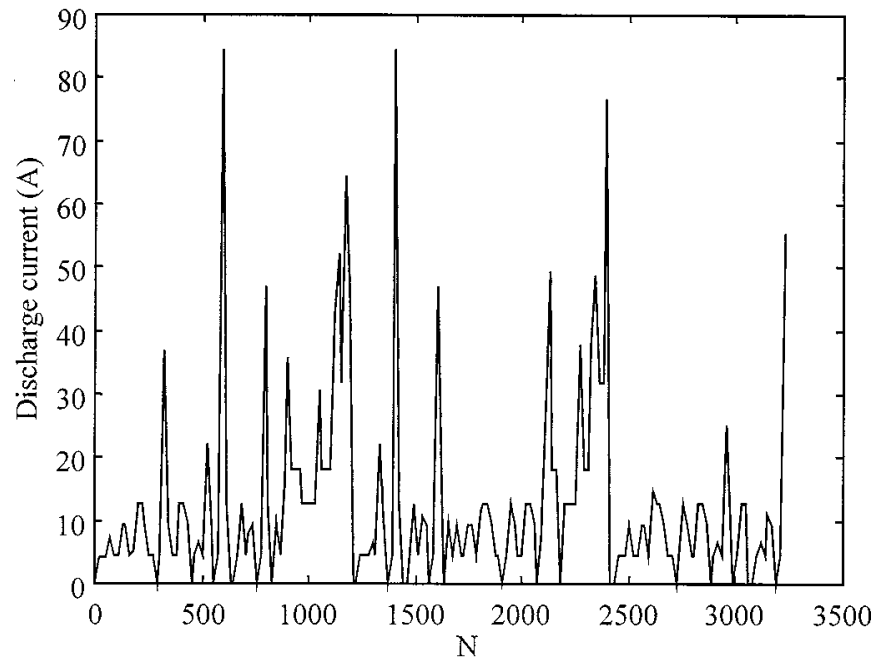

(a)

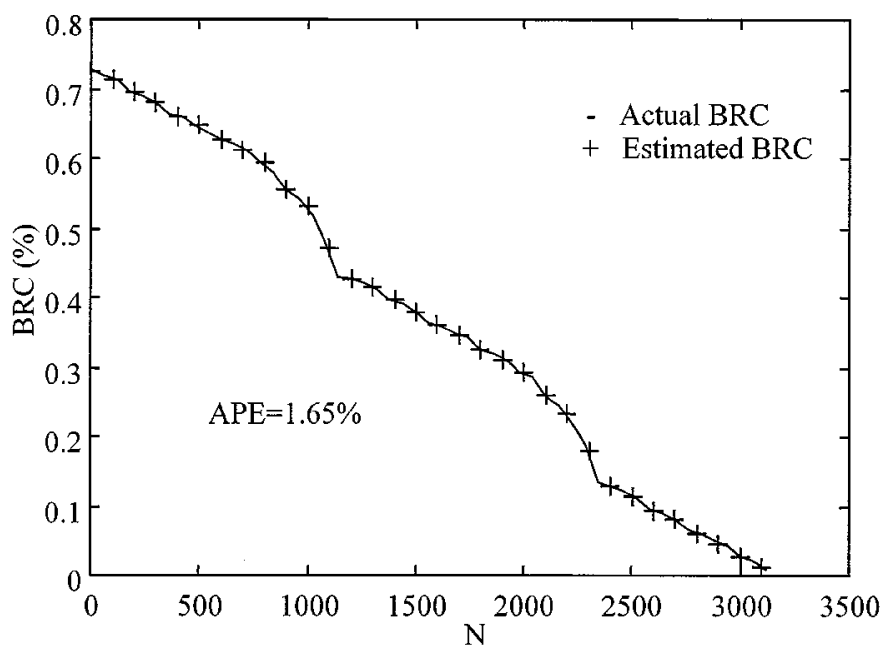

(b)

Fig. 7. Comparison between actual BRC and estimated BRC for ECE operation. (a) ECE discharge profile. (b) BRC profiles.

trained ANFIS model and the actual BRC from the experimental data, respectively. The APEs for both the training data set and the data set of each test are calculated. Fig. 4 shows the estimated $\mathrm{BRC}$ and the actual BRC for the training data set. It can be found that the estimation is of high accuracy, and the corresponding APE is only $0.53 \%$.

To evaluate the trained ANFIS model for the estimation of the BRC effectively, the 17 data sets for each test are used to verify the trained model. The results corresponding to the aforementioned CCD, RCD, ECE, FUDS, and JM10.15 operations are shown in Figs. 5-9, respectively. All these figures illustrate that the proposed method provides highly accurate estimation of the BRC for different operating profiles of EVs. It should be noted that the APEs of the proposed ANFIS model are all within 2\%, which offers a significant improvement over the APE of $10 \%$ in [15] in which the ANN model is adopted.

The ANFIS model can estimate the BRC accurately due to two facts. First, the ANFIS model inherently offers a merit to ac-

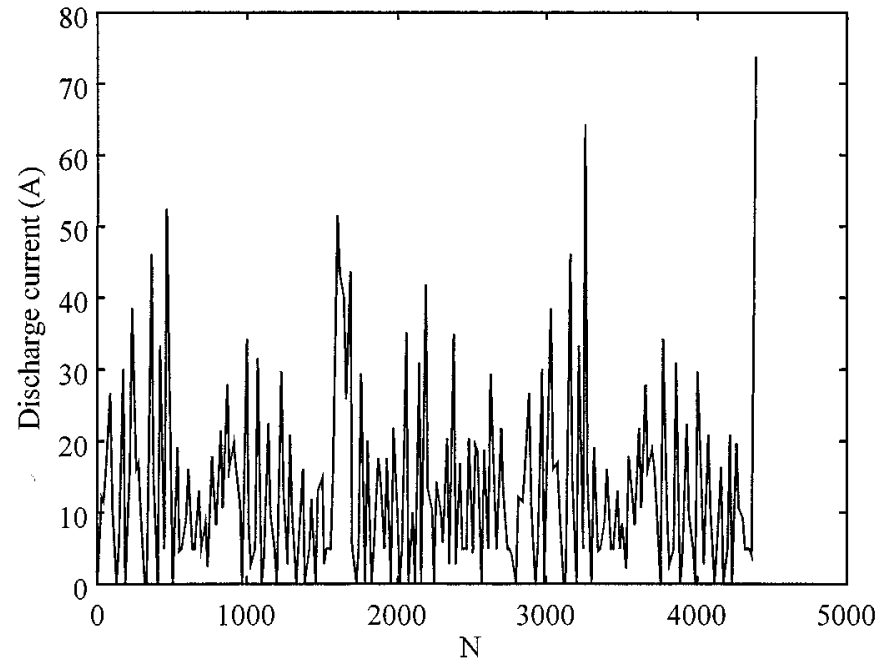

(a)

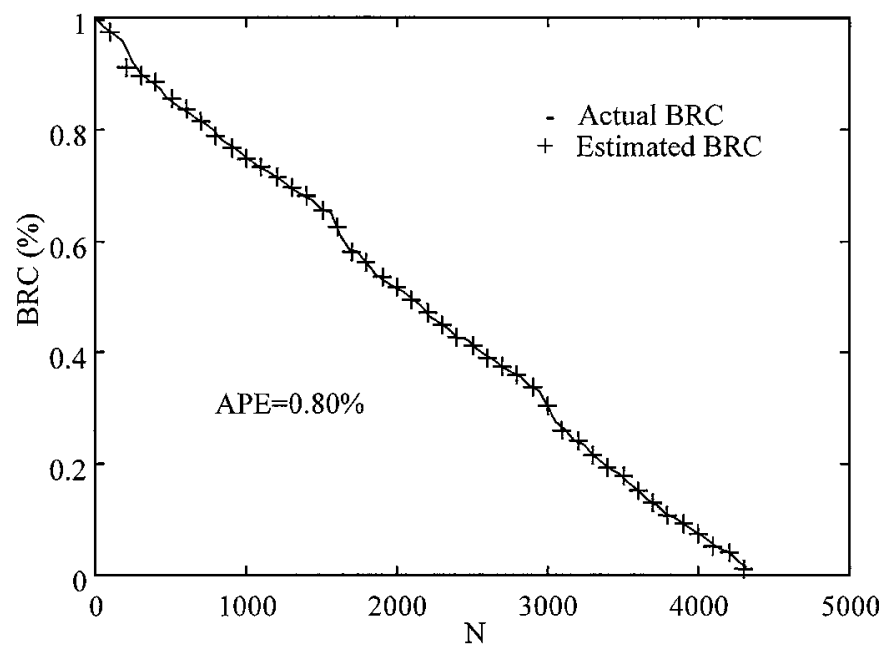

(b)

Fig. 8. Comparison between actual BRC and estimated BRC for FUDS operation. (a) FUDS discharge profile. (b) BRC profiles.

curately represent complex nonlinear mappings. The nonlinear mapping from the battery terminal voltage, the discharge current, the discharged capacity, and the battery surface temperature to the BRC under their practical operating ranges can fully utilize this inherent merit. Second, the proposed data selection procedure of the training data set can enable the ANFIS model minimizing the error between the estimated BRC and the actual BRC while maintaining its generality. Thus, it is expected that the model can accurately represent the battery characteristics for the purpose of BRC estimation. On the other hand, this ANFIS model potentially offers high adaptability provided that it can be regularly retrained by new training data sets. The keys are how to extract the informative data from those new discharge current profiles and how to identify the obsolete data from those previous discharge current profiles so that the training data set can be effectively updated. The corresponding investigation will be our future work in the area of ANFIS modeling for the BRC estimation. 


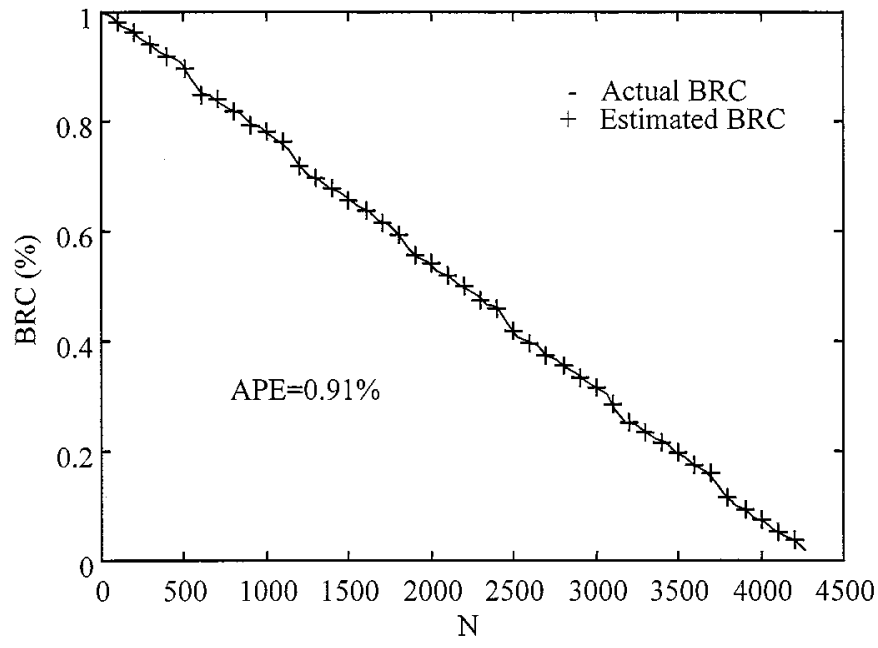

(a)

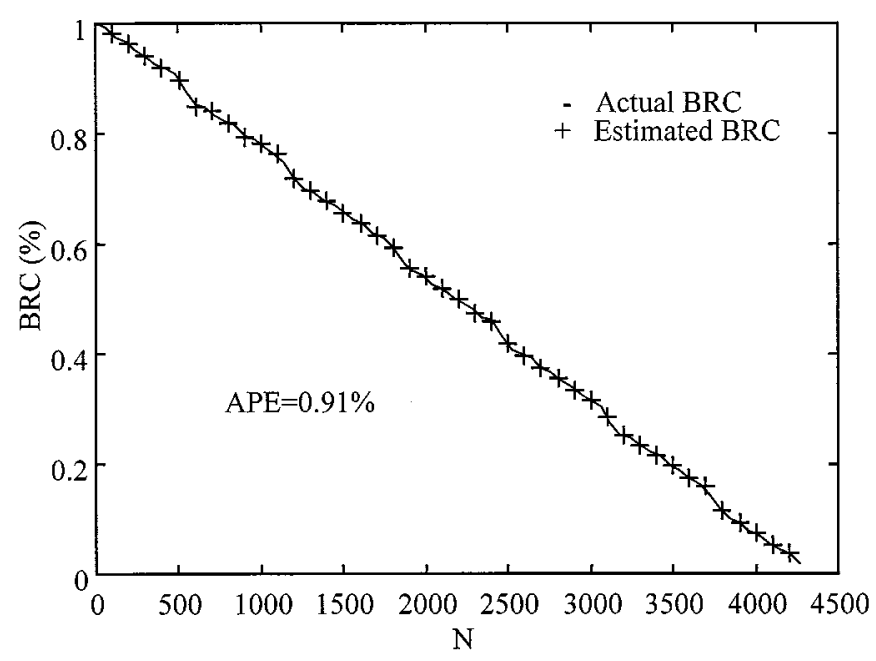

(b)

Fig. 9. Comparison between actual BRC and estimated BRC for JM10.15 operation. (a) JM10.15 discharge profile. (b) BRC profiles.

The proposed ANFIS model for the BRC estimation in EVs can also be easily implemented by a low-cost microcontroller (such as the 80C96), which is illustrated in Fig. 10. The reason is that the BRC estimation only involves the basic operation of addition, subtraction, multiplication, and division after the premise and consequent parameters of the ANFIS model are obtained. In contrast, the implementation of the ANN model involves the exponential calculation which is difficult to be realized by a low-cost microcontroller.

\section{CONCLUSIONS}

This paper has proposed and implemented the application of the ANFIS model to the BRC estimation for EVs. Comparisons between the estimated results from the proposed model and the experimental data show good agreement, verifying its accuracy and effectiveness for the estimation of the BRC. Also, this new estimation method can easily be implemented by a low-cost mi-

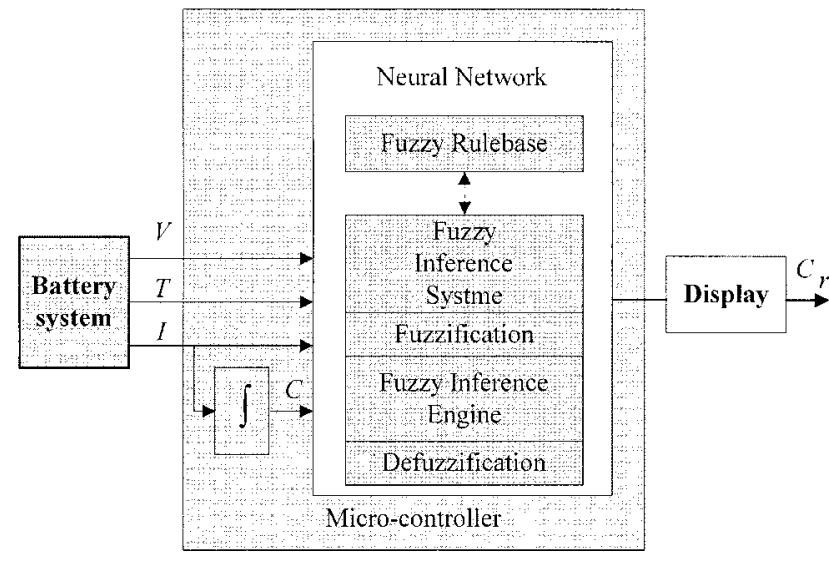

Fig. 10. Implementation diagram of the BRC estimator.

crocontroller and readily be used for the BRC estimation in EVs. Finally, the proposed method is so general that it can be extended to the BRC estimation for other types of EV batteries.

\section{REFERENCES}

[1] C. C. Chan, "An overview of electric vehicle technology," Proc. IEEE, vol. 81, pp. 1202-1213, Sept. 1993.

[2] C. C. Chan and K. T. Chau, "An overview of power electronics in electric vehicles," IEEE Trans. Ind. Electron., vol. 44, pp. 3-13, Feb. 1997.

[3] C. C. Chan, E. W. C. Lo, and W. X. Shen, "The overview of battery technology in electric vehicles," in Proc. Int. Electric Vehicle Symp., 1999, CD-ROM.

[4] R. Kaushik and I. G. Mawston, "Discharge characterization of lead-acid batteries," J. Power Sources, vol. 28, no. 1, pp. 161-169, 1989.

[5] S. K. Song and K. H. Kim, "A dynamic state of charge model for electric vehicles batteries," in Proc. Int. Electric Vehicle Symp., vol. 2, 1994, pp. 519-527.

[6] J. F. Riutort, E. Rulliere, and E. Toutain, "An improved coulometric gauge for electric vehicles," in Proc. Electric Vehicle Technology Conf., vol. 3, 1995, pp. 7-15.

[7] O. Caumont, P. Le Moigne, C. Rombaut, X. Muneret, and P. Lenain, "Energy gauge for lead-acid batteries in electric vehicles," IEEE Trans. Energy Conversion, vol. 15, pp. 354-360, Sept. 2000.

[8] Z. M. Salameh, M. A. Casacca, and W. A. Lynch, "A mathematical model for lead-acid batteries," IEEE Trans. Energy Conversion, vol. 7, pp. 93-98, Mar. 1992.

[9] D. Fan and R. E. White, "Mathematical modeling of a nickel-cadmium battery-effects of intercalation and oxygen reactions," J. Electrochem. Soc., vol. 138, no. 10, pp. 2952-2960, 1991.

[10] H. J. Bergveld, W. S. Kruijt, and P. H. L. Notten, "Electronic-network modeling of rechargeable NiCd cells and its application to the design of battery management system," J. Power Sources, vol. 77, no. 2, pp. 143-158, 1999.

[11] J. H. Aylor, A. Thieme, and B. W. Johnson, "A battery state-of-charge indicator for electric wheelchairs," IEEE Trans. Ind. Electron., vol. 39, pp. 398-409, Oct. 1992.

[12] E. Karden, P. Mauracher, and F. Schoepe, "Electrochemical modeling of lead-acid batteries under operating conditions of electric vehicles," $J$. Power Sources, vol. 64, no. 1, pp. 175-180, 1997.

[13] E. Barsoukov, J. H. Kim, C. O. Yoon, and H. Lee, "Universal battery parameterization to yield a nonlinear equivalent circuit valid for battery simulation at arbitrary load," J. Power Sources, vol. 83, no. 1-2, pp. 61-70, 1999.

[14] J. C. Peng, Y. B. Chen, R. Eberhart, and H. H. Lee, "Adaptive battery state of charge estimation using neural network," in Proc. Int. Electric Vehicle Symp., 2000, CD-ROM.

[15] T. Yamazaki, K. Sakurai, and K. I. Muramoto, "Estimation of the residual capacity of sealed lead-acid batteries by neural network," in Proc. Int. Telecommunications Energy Conf., vol. 20, 1998, pp. 210-214.

[16] C. C. Chan, E. W. C. Lo, and W. X. Shen, "Available capacity computation model based on artificial neural network for lead-acid batteries in electric vehicles," J. Power Sources, vol. 87, no. 1-2, pp. 201-204, 1999. 
[17] _ - "A new calculation approach of the available capacity of batteries in electric vehicles," in Proc. Int. Electric Vehicle Symp., 2000, CD-ROM.

[18] A. J. Salkind, C. Fennie, P. Singh, T. Atwater, and D. E. Reisner, "Determination of state of charge and state of health of batteries by fuzzy logic methodology," J. Power Sources, vol. 80, no. 1-2, pp. 293-300, 1999.

[19] C. T. Lin and C. S. G. Lee, Neural Fuzzy Systems. Englewood Cliffs, NJ: Prentice-Hall, 1996.

[20] X. J. Zeng and M. G. Singh, "Approximation accuracy analysis of fuzzy systems as function approximators," IEEE Trans. Fuzzy Syst., vol. 4, pp. 44-63, Feb. 1996.

[21] J. R. Jang, "ANFIS: Adaptive-network-based fuzzy inference system," IEEE Trans. Syst., Man, Cybern., vol. 23, pp. 665-685, May/June 1993.

[22] — " "Input selection for ANFIS learning," Proc. IEEE Int. Conf. Fuzzy Systems, vol. 2, no. 1, pp. 1493-1499, 1996

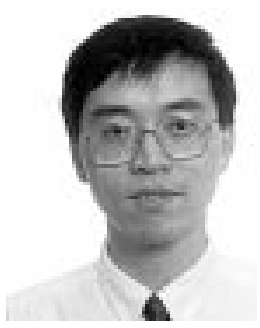

W. X. Shen (S'00) received the M.Sc.(Eng.) degree in automatic control from Shanghai Jiaotong University, Shanghai, China, in 1990. He is currently working toward the Ph.D. degree at The University of Hong Kong, Hong Kong.

In 1990, he joined the Department of Electrical Engineering, Hefei University of Technology, Hefei, China, where he has been an Associate Professor since 1995. From 1993 to 1994, he was a Visiting Scholar at the University of Stuttgart, Stuttgart, Germany. His research interests include battery modeling technology, charging algorithms, and system simulations for electric vehicles and photovoltaic systems.

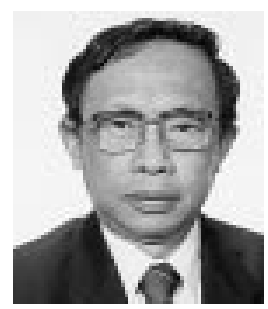

C. C. Chan (M'77-SM'77-F'92) received the B.Sc. degree from China University of Mining and Technology, Beijing, China, the M.Sc. degree from Tsinghua University, Beijing, China, and the Ph.D. degree from The University of Hong Kong, Hong Kong, in 1953, 1957, and 1981, respectively.

$\mathrm{He}$ is currently Chair Professor in the Department of Electrical and Electronic Engineering, The University of Hong Kong. He has authored four books and more than 120 published technical papers and is the

holder of seven patents.

Prof. Chan is a Fellow of the Royal Academy of Engineering, U.K., an Academician of the Chinese Academy of Engineering, and a Fellow of the Ukraine Academy of Engineering Science. He was awarded the Honorary D.Sc. degree from the University of Odessa in 1992.

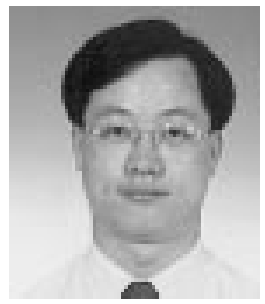

E. W. C. Lo (M'83) received the B.Sc. (Eng.), M.Phil., and Ph.D. degrees from The University of Hong Kong, Hong Kong, in 1983, 1986, and 1996, respectively, all in electrical and electronic engineering.

Since 1995, he has lectured in the Department of Electrical and Electronic Engineering, The University of Hong Kong. He previously held a number of academic posts at other universities in Hong Kong and spent a period in industry with Telstra, Ltd., Australia. His current research interests include drives and traction, electric vehicles, electrical installations in buildings, and renewable energy.

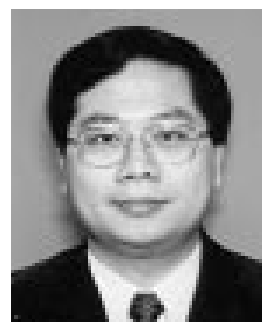

K. T. Chau ( $\left.\mathbf{M}^{\prime} 89\right)$ received his first-class honors B.Sc.(Eng.), M.Phil., and Ph.D. degrees in electrical and electronic engineering from The University of Hong Kong, Hong Kong.

He is currently an Associate Professor at The University of Hong Kong. His teaching and research interests focus on three main areas: power converters, machines and drives, and electric vehicles. In these areas, he has authored more than 100 published refereed technical papers and several industrial reports. He has also served as chair and organizing committee member for many international conferences. He is the coauthor of a monograph, Modern Electric Vehicle Technology (London, U.K.: Oxford Univ. Press, 2001). 